\title{
Supplements: Distilling Knowledge in Causal Inference for Unbiased Visual Question Answering
}

\author{
Yonghua Pan \\ yonghuapan@njust.edu.cn \\ Nanjing University of Science \& Technology \\ Nanjing, China \\ Liyan Zhang \\ zhangliyan@nuaa.edu.cn \\ Nanjing University Of Aeronautics \& Astronautics \\ Nanjing, China
}

\section{ACM Reference Format:}

Yonghua Pan, Zechao Li, Liyan Zhang, and Jinhui Tang. 2021. Supplements: Distilling Knowledge in Causal Inference for Unbiased Visual Question Answering. In ACM Multimedia Asia (MMAsia '20), March 7-9, 2021, Virtual Event, Singapore. ACM, New York, NY, USA, 2 pages. https://doi.org/10.1145/ 3444685.3446256

\section{DETAILS OF CAUSAL GRAPH}

\subsection{An Example for Building A Causal Graph}

The causal graph is used to model causal assumption [3]. In this section, an example is given to well explain the process of building a causal graph. Let $U$ be a set of exogenous variables, $V$ be the set of endogenous variables, and $F$ be a set of functions. The formal expressions of variables and functions are as follows.

$$
\begin{array}{r}
U=\{X, Y\}, V=\{Z\}, F=\left\{f_{Z}\right\} \\
f_{Z}: Z=2 X+3 Y
\end{array}
$$

The causal graph associated with Eq. (1) and Eq. (2) is illustrated in Figure 1.

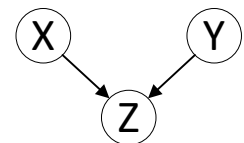

Figure 1: The example of the causal graph associated

Link $X \rightarrow Z$ and $Y \rightarrow Z$ are caused by the function $f_{Z}$. This is because the function $f_{Z}$ obtains $Z$ through $X$ and $Y$.

*corresponding author.

Permission to make digital or hard copies of all or part of this work for personal or classroom use is granted without fee provided that copies are not made or distributed for profit or commercial advantage and that copies bear this notice and the full citation on the first page. Copyrights for components of this work owned by others than ACM must be honored. Abstracting with credit is permitted. To copy otherwise, or republish, to post on servers or to redistribute to lists, requires prior specific permission and/or a fee. Request permissions from permissions@acm.org.

MMAsia '20, March 7-9, 2021, Virtual Event, Singapore

(c) 2021 Association for Computing Machinery.

ACM ISBN 978-1-4503-8308-0/21/03 . \$ \$15.00

https://doi.org/10.1145/3444685.3446256

\author{
Zechao $\mathrm{Li}^{*}$ \\ zechao.li@njust.edu.cn \\ Nanjing University of Science \& Technology \\ Nanjing, China \\ Jinhui Tang \\ jinhuitang@njust.edu.cn \\ Nanjing University of Science \& Technology \\ Nanjing, China
}

\subsection{Causal Graph of The VQA Model}

As introduced in 1.1, the causal graph of the VQA model is built by the calculation process of the UpDn model [1]. The meanings of the causal relations and nodes are as follows.

Node $Q$ (Question). The embeddings of words in questions are initialized by GloVe [4]. GRU [2] is utilized as the question encoder to encode the question $q_{i}$ to a vector $f_{i}^{q}$.

Node $V$ (Object feature). The exogenous variable of Node $V$ is the visual features of detected object regions $\boldsymbol{o}_{i}=\left\{f_{i, m}^{v}\right\}_{m \in[1: M]}$. The object feature $o_{i}$ is extracted by a pre-trained and frozen Faster R-CNN [5], where $\boldsymbol{o}_{i}$ indicates the object features of the $i$-th sample, $M$ indicates the number of region, $f_{i, m}^{v}$ indicates the object feature extracted from the $m$-th region of the $i$-th sample. The variable of node $V$ denotes the visual feature obtained by the question-guided attention mechanism.

Node $B$ (Bias learned from data). The conventional VQA model learn the data bias between questions and answers. The bias in the conventional VQA model is a mediation between $Q$ and $A$ (i.e., $Q \rightarrow B \rightarrow A$ ). It is worth noting that it is hard to confirm which features in the question leads to the model bias since unbiased VQA data is difficult to be obtained.

Link $Q \rightarrow V$ (Attention mechanism). Question-guided attention $\boldsymbol{\alpha}_{i} \in \mathbb{R}^{M}$ is formulated as $\boldsymbol{\alpha}_{i}=\operatorname{att}\left(\operatorname{repeat}\left(f_{i}^{q}\right) \odot \boldsymbol{o}_{i}\right)$, where $\alpha_{i}$ indicates the attention of the $i$-th sample, $\odot$ denotes the elementwise product, att is the fully connected layer, repeat $\left(f_{i}^{q}\right)$ is used to repeat $f_{i}^{q} M$ times and stack the repeated vector to a matrix. And the $m$-th region visual feature $f_{i, m}^{\alpha}$ is calculated by $f_{i, m}^{\alpha}=\alpha_{i, m} * f_{i, m}^{v}$, $\alpha_{i, m}$ is the $m$-th element of $\alpha_{i}$.

Link $Q \rightarrow B$ (Bias caused by question). The Q-only model has good performance on the standard VQA datasets since the question encoder learns the statistical correlation between questions and answers. Therefore, the question encoder in VQA models may cause the bias in model.

Link $\{Q, V, B\} \rightarrow A$ (Answer classification). We introduce sumpooling to fuse visual features $\left\{f_{i, m}^{\alpha}\right\}_{m \in M}$. The answer classifier is formulated as $\hat{\boldsymbol{p}}_{i}=\operatorname{softmax}\left(f_{i}^{q} \odot \sum_{m}^{M} f_{i, m}^{\alpha}\right)$, where $\hat{\boldsymbol{p}}_{i} \in \mathbb{R}^{K}$ is the confidence of candidate answers. Due to $f_{i}^{q}$ and $f_{i, m}^{\alpha}$ come from the encoding feature of the observed values of $Q$ and $V$ respectively, causality relation $\{Q, V\} \rightarrow A$ is defined. Since the biases come from the question feature $f_{i}^{q}$, causality relation $B \rightarrow A$ is defined. 
Node $A$ (Predicted answer). The final predicted answer is generated by the answer classifier based on the inputs from three branches.

\section{REFERENCES}

[1] Peter Anderson, Xiaodong He, Chris Buehler, Damien Teney, Mark Johnson Stephen Gould, and Lei Zhang. 2018. Bottom-Up and Top-Down Attention for Image Captioning and Visual Question Answering. In Proceedings of IEEE Conference on Computer Vision and Pattern Recognition. 6077-6086.
[2] Junyoung Chung, Caglar Gülçehre, KyungHyun Cho, and Yoshua Bengio. 2014. Empirical Evaluation of Gated Recurrent Neural Networks on Sequence Modeling. arXiv preprint arXiv:1412.3555 (2014).

[3] Judea Pearl, Madelyn Glymour, and Nicholas P Jewell. 2016. Causal Inference in Statistics: A Primer. John Wiley \& Sons.

[4] Jeffrey Pennington, Richard Socher, and Christopher Manning. 2014. GloVe: Global Vectors for Word Representation. In Conference on Empirical Methods in Natural Language Processing. 1532-1543.

[5] Shaoqing Ren, Kaiming He, Ross Girshick, and Jian Sun. 2017. Faster R-CNN: Towards Real-Time Object Detection with Region Proposal Networks. IEEE Trans. on Pattern Analysis and Machine Intelligence 39, 6 (2017), 1137-1149. 